\title{
Experience of nursing students about the barriers to patient education: a qualitative study in Iran
}

\author{
Mohammad Abbasi ${ }^{1}$, Leili Rabiei ${ }^{2}$ and Reza Masoudi ${ }^{3}$ \\ ${ }^{1}$ Department of Nursing, School of Nursing and Midwifery, Oom University of Medical Sciences, Qom, ${ }^{2}$ Social \\ Determinants of Health Research Center, Shahrekord University of Medical Sciences, and ${ }^{3}$ Community Oriented \\ Nursing Midwifery Research Center, Shahrekord University of Medical Sciences, Shahrekord, Iran
}

Purpose: Patient education is a dynamic and continuous process that should be implemented during the entire time of hospital stay and even afterward. Studies have shown the typically poor quality of patient education in Iran and its failure to convey the required knowledge and skills to patients. The purpose of this study was to survey the experience of nursing students in regard to the challenges of patient education in hospitals.

Methods: This qualitative study was conducted using the conventional qualitative content analysis approach on a sample of 21 undergraduate nursing students (4th semester and beyond), which was drawn from the Oom Nursing and Midwifery School through purposive sampling with maximum variation. Data were collected through semi-structured interviews conducted over a period of 45 to 75 minutes, and were analyzed using the conventional qualitative content analysis.

Results: Results were derived from the experiences of 21 nursing students (nine males, 12 females) about the research subject. The primary themes identified in the study were the student-related, patient-related, instructor-related, education environment-related, and curriculum-related barriers to patient educations.

Conclusion: Participants believed that patient education in Iranian hospitals is faced with many challenges. Nursing instructors and curriculum planners should ensure more emphasis on patient education at the initial semesters of nursing education curriculum and make sure that it is included in the evaluation of students. Hospital officials should provide a dedicated education environment with suitable facilities, tools, and atmosphere for patient education. Also, special education programs need to be developed for less educated patients.

Key Words: Patient education as topic, Nursing students, Qualitative research, Iran

\section{Introduction}

Patient education is a dynamic and continuous process that should be carried out over the entire time from the patient admission to discharge from hospital. Patient education improves the knowledge, skills, and motiva- tion of patients about maintaining and improving their health. Patient education is known to improve patient satisfaction and quality of life, reduce the incidence of complications, cost of treatment, and rate of readmission [1-5], reinforce healthy behaviors and reduce unhealthy behaviors, reduce patient anxiety, decrease the effect of symptoms, shorten the hospital stay time, increase the
Received: February 13, 2018 • Revised: March 16, 2018 • Accepted: May 13, 2018 Corresponding Author: Reza Masoudi (https://orcid.org/0000-0003-3534-0550) Department of Nursing, School of Nursing and midwifery, Shahrekord University of Medical Sciences, Shahrekord, IR Iran

Tel: +98.38.33335648 Fax: +98.38.33336699 email: masoodi1383@yahoo.com
Korean J Med Educ 2018 Dec; 30(4): 327-337.

https://doi.org/10.3946/kjme.2018.107

eISSN: 2005-7288

(C) The Korean Society of Medical Education. All rights reserved. This is an open-access article distributed under the terms of the Creative Commons Attribution Non-Commercial License (http:// creativecommons.org/licenses/by-nc/3.0/), which permits unrestricted non-commercial use, distribution, and reproduction in any medium, provided the original work is properly cited. 
participation in health care programs, and improve the patient autonomy in daily activities [6]. Studies have shown that patient education efforts are of poor quality and fail to convey the necessary knowledge and skills to patients $[7,8]$. According to the opinions of Edwardson [9], patient education performance of nurses is unsatisfactory, and Deccache and Aujoulat [10] report that only a small number of patients believe they receive sufficient information and counseling [11].

On the other hand, nurses have expressed that there are many challenges ahead of the successful implementation of patient education. Ghorbani et al. [7] report that one of such challenges is the deficiency of educational tools such as patient education leaflets. The crucial point is this fact that the lack of awareness of the necessity of patient education in clinical practice and theory is the most important fundamental challenge. Lack of awareness of the importance of patient education on the part of practicing nurses, students, and teachers is quite tangible. Some of these barriers are mentioned in a few studies; According to studies of Tahery et al. [8], students believe that the most important obstacles to patient education are the physical and mental problems of patients, the insufficient knowledge and skills of nurses about the diseases, the small number of the hospital nurses, and the lack of trust between the patient and the educator.

Nursing students, as the future nurses, will have a great role in the future of patient education, and are shown to have a positive attitude toward this issue. But in practice, patient education is not satisfactorily at the moment [12]. Identification of the factors that undermine patient education can be a positive step toward the provision of better patient education by future nurses [6,12-14]. According to these studies, surveying the opinions and experience of nursing students about the barriers to patient education may contribute to the resolution of this issue through careful planning and formulation of specific strategies in the future. All of the previous studies in this area have followed a quantitative approach and just intermittently highlighted some of the obstacles to patient education. Identification of these barriers and the related hidden layers from the experiences of nursing students through a qualitative approach can provide valuable insight into this subject. Therefore, this study aimed to survey the experiences of nursing students in regard to the barriers to patient education.

\section{Methods}

\section{Subjects}

The goal of this qualitative study was to survey the experiences of nursing students in regard to the barriers to patient education. This goal was pursued using the conventional content analysis method, which is the subjective interpretation of data from a body of text through the analysis and categorization of themes in the content [11].

The research was conducted in the Nursing and Midwifery School of the Qom University of Medical Sciences in 2017. The subjects were selected through purposive sampling with maximum variation. For this purpose, nursing students at the Nursing and Midwifery School were invited to enroll in the research project. The criteria for enrollment were: being a nursing student in 4th semester and beyond and willingness to participate in research and express their experiences. Data was collected through in-deep semi-structured interviews. Before the interviews, the participants were informed about the purpose of the study, asked to allow the interview to be recorded, and ensured about the 
confidentiality of the interview and all personal information, and then asked to give verbal informed consent. Participants were allowed to leave the research at will and expressed their experiences voluntarily. After recording the demographic characteristics, participants were asked to express their experiences about the barriers to patient education. The interviews started by asking the questions as: "Please talk about the events of a regular day of internship at the hospital," "Please talk about what you do while attending the patients," "Please talk about the patient education," and "Please talk about the barriers to patient education." When needed, further inquiries about remarks were made using the questions and request such as: "Please explain more about what you said" or "What did you mean when you said..." Each interview took between 45 and 75 minutes. The interviews were conducted in Persian by the first author, recorded and transcribed verbatim, and then translated into English.

\section{Data analysis}

Data were analyzed using the conventional content analysis in eight stages to identify the barriers to patient education. For this purpose, the interviews were first converted into text. The entire text of each interview was then reviewed and the semantic units (words, sentences, or paragraphs describing the barriers to patient education) were listed. After coding and reviewing the text derived from the interviews (with the semantic units converted to more general concepts and phrases), the codes were integrated based on similarities and differences, and another classification was performed to extend the categories based on their similarity and relation. The categories were again checked with the data to ensure the strength of the codes for theme identification. Finally, the results regarding the barriers to patient education were obtained through careful inference and comparison of categories with each other. The accuracy, validity, and reliability of the data obtained from this qualitative approach were investigated using the criteria specifically proposed for this purpose, i.e., credibility, dependability, confirmability, and transferability [15]. To enhance the credibility of the study, the researcher remained engaged with the issue for a prolonged duration. The data obtained from interviewed were also reviewed and confirmed by the participants, who then provided some feedback regarding the data. Critical and complementary comments of colleagues and peers were also taken into consideration. The dependability of data was evaluated using the stepwise replication and audit trail methods. This was done by presenting the results to the respected referee of the research project. To ensure the confirmability of findings, all stages of the research were accurately recorded and confirmed by a third party with expertise on qualitative research. To ensure the transferability, the researcher attempted to provide the exact remarks of participants and also tried to provide further details about the characteristics of the participants and the research environment to facilitate future works and the use of the results in other studies with other contexts.

\section{Ethical considerations}

The ethical approval was obtained from Research and Technology Deputy of Qom University of Medical Sciences for all the four healthcare centers under study (ethical code: IR.MUQ.REC.1396.30.). In this study, the participants were informed of the purposes and significance of the research and then the written informed consent to participate in the study was obtained from them. Also, the participants were assured that participation in the study is voluntary and can be discontinued at any time. The participants were aware of the purpose and importance of research and informed written consent 
was obtained. Also participants were assured that participation in the study was voluntarily and that they may withdraw from the study at any time.

\section{Results}

The sample population consisted of 21 nursing students (nine males and 12 females) at the Nursing and Midwifery School of Qom University of Medical Sciences. The texts obtained from the conversion of audio files of interviews were subjected to in-depth analysis, which produced 575 initial codes. The codes were reviewed several times and classified based on similarity. Barriers to patient education in view of nursing students were found in five themes: (1) student-related barriers: student's lack of motivation, willingness, and patience, student's inability to express academic knowledge in simple words for patient to understand, and student's lack of sufficient academic knowledge to educate the patients, fatigue; (2) patient-related barriers: patient's lack of demand to receive education, patient's severe illness, patient's lack of education, patient's inability to communicate with the student, the cultural barriers between the patient and the student, patient's refusal to accept the student, patient's poor opinion toward the students, and patient's lack of interest in provided knowledge; (3) instructor-related barriers: instructors' failure to give patient education assignments to the students, instructor's focus on clinical proficiency, instructor's disregard for the relationship between the student and the patient, instructor's lack of interest or concern about the patient education, the lack of division of labor in the care process, and instructor's emphasis on the routine work; (4) education environment-related barriers: wrong clinical practices, high workload, personnel's disinterest in patient education, the lack of education tools, facilities, and atmosphere, and physicians and head nurses' disregard for patient education; and (5) curriculum-related barriers: inattention to $\mathrm{pa}^{-}$ tient education in the current curriculum and absence of patient education from the listed duties of nursing students (Table 1).

\section{Student-related barriers}

All participants pointed out the role of students in the deficiency of patient education. They talked about the students' lack of interest, motivation, and patience as well as their fatigue. They also stated that students do not have sufficient academic knowledge to educate the patient, and when they do have such knowledge, they are often unable to explain it in plain language.

In this regard, one of the participants stated: "When I go to a patient and try, for example, to explain how to prepare or inject an insulin shot, I just can't do it" (participant No. 11).

Some participant talked about their reluctance and lack of willingness and motivation to educate patients. Some of the nursing students who had part-time jobs or other commitments also said that they do not have the energy or willingness to educate the patients.

In this regard, one of the participants stated: "I work at nights to financially support myself. In the morning, when I go to my internship, I'm so tired that I barely make it to the noon, so educating the patients is out of the question" (participant No. 4).

Some participant stated that they lack the sufficient academic knowledge or mastery over the subjects for educating the patients.

A participant stated: "Unfortunately, I have a poor academic performance so I can't go to the patient and comfortably explain even the simplest of notions" (participant No. 7).

In this regard, another participant stated: "When I 
Table 1. Primary and Secondary Themes of Barriers to Patient Education

\begin{tabular}{cc}
\hline \multicolumn{1}{c}{ Primary themes } & \multicolumn{1}{c}{ Secondary themes } \\
\hline $\begin{array}{c}\text { Student- } \\
\text { related barriers }\end{array}$ & $\begin{array}{c}\text { Lack of motivation and willingness, inability } \\
\text { to express academic knowledge in simple } \\
\text { words, lack of sufficient academic } \\
\text { knowledge }\end{array}$ \\
Patient-related barriers & $\begin{array}{c}\text { Patients not demanding any education, } \\
\text { patient's severe illness, patient's lack of } \\
\text { education, patient's inability to } \\
\text { communicate with the student, cultural } \\
\text { barriers between the patient and the } \\
\text { student, patient's refusal to accept the } \\
\text { student, patient's poor opinion toward } \\
\text { the student, patient's lack of interest }\end{array}$
\end{tabular}

Instructor-

related barriers

Instructors making no demand in regard to patient education, instructors' focus on clinical work and inattention to the relationship between the student and the patient, instructor's lack of interest or concern regarding the patient education, absence of division of labor, instructors' emphasis on the routine work

Education environment- Wrong clinical practices, high workload, related barriers personnel's disinterest, lack of education tools, facilities and atmosphere, failure of physicians and head nurses to give patient education assignments to the nursing students

curriculumrelated barriers
Inattention to patient education in the curriculum, absence of patient education from the listed duties of nursing students
Examples of comments

"I'm not interested in educating the patient." "I can't teach the patient in a simple and understandable language." "Unfortunately, I have a poor academic performance so I can't go to the patient and comfortably explain even the simplest of notions."

"Sometimes I want to give an explanation about a medicine, for example, but I see that patient doesn't look interested; some patients don't even listen." "One of the problems with teaching the patients is that they are in a bad state." "Many hospitalized patients can't communicate with hospital personnel." "The place where I do my internship, patients are coming from many different parts of the country, and sometimes I just can't comprehend their language and dialect; some patients don't even listen when we talk." "When a patient is illiterate, you can't explain even the simplest things. I always try my best to do it, but it's very hard." "Many patients refuse to be cared by a nursing student; some even refuse to let us measure their blood pressure, let alone educate them." "Many patients aren't interested in receiving education; when we talk to them about the disease or a drug, they just don't seem to care."

"In the mornings, when we enter the ward, it seems that many of our instructors don't have a specific program for the day; we usually follow a routine, and instructors don't ask us to educate the patients." "Most of our instructors are occupied with routine work; educating the patients isn't a part of our daily work, so we don't do it, because they don't ask us to do it." "In many wards, we don't have a specific program. In the morning, we go to the ward and start doing routine work. Many instructors just want us to have physical presence in the ward and don't care what we do until noon, and the same goes for patient education."

"Even if somebody wants to educate the patient, there's no place to do this; there must some place to do this, and some tools; maybe a patient doesn't like to be educated about his condition in front of other patients." "The wards are so busy and the workload is so high that we even struggle to follow our routines and respond to the clinical needs of patients." "Teaching the patient isn't a priority in any ward; so far, I haven't seen any ward putting this in its protocol; even doctors and nurses themselves don't take this seriously."

"We only had one course about patient education, and I don't think that even that was about educating patients. If patient education is so important, why they don't teach us anything about it." "Patient education isn't in the list of duties of nursing students; so we aren't obliged to do it." control a patient's blood pressure, I'm always afraid that what if he asks me a question; I have a lot of trouble dealing with the examiners" (participant No. 14).

\section{Patient-related barriers}

Many participants stated that patients are not demanding or willing to receive any education. They also said that patients were generally not interested in the description of the drugs.
In this regard, one of the participants stated "Sometimes I want to give an explanation about a medicine, for example, but I see that patient doesn't look interested; some patients don't even listen" (participant No. 14).

Some participants argued that the patient's state of illness and inability to properly communicate were other barriers to patient education.

A participant stated: "One of the problems with teaching the patients is that they are in a bad state" 
(participant No. 13).

Another participant said: "Many hospitalized patients can't communicate with hospital personnel" (participant No. 19).

Many participants believed that another important barrier to patient education is the cultural barrier, which is the difference between the language or dialect of the patient and that of the student. In regard to this barrier, one participant said: "The place where I do my internship, patients are coming from many different parts of the country, and sometimes I just can't comprehend their language and dialect; some patients don't even listen when we talk" (participant No. 18).

All participants highlighted the patients' illiteracy and low education as one of the barriers to patient education. One of the participants stated: "When a patient is illiterate, you can't explain even the simplest things. I always try my best to do it, but it's very hard" (contributor No. 18).

Many participants believed that patients did not have a favorable opinion toward the nursing students and see their work with distrust, even when the procedure is performed under direct supervision of an instructor. "Many patients refuse to be cared by a nursing student; some even refuse to let us measure their blood pressure, let alone educate them" said one participant (participant No. 8).

The patients' disinterest in receiving an education was another obstacle. One participant stated: "Many patients aren't interested in receiving education; when we talk to them about their disease or a drug, they just don't seem to care" (participant No. 18).

\section{Instructor-related barriers}

One of the barriers to patient education was found to be the instructors' performance and preferences in planning the students' daily clinical work program. The participants of this study stated that many clinical nursing instructors did not pay any attention to patient education when planning the daily programs and schedules. According to the participants, instructors' failure to set patient education objectives for nursing students, their focus on the routine clinical work and the physical presence in the ward, their disregard for the concept of patient education, the absence of division of labor, and opaqueness of the daily work assignments are some of the issues that obstruct the provision of patient education by nursing students.

Many participants stated that patient education is not on their daily schedule. One participant said: "In the mornings, when we enter the ward, it seems that many of our instructors don't have any specific plan for the day; we usually follow a routine, and instructors don't ask us to educate the patients" (participant No. 11).

In regard to daily routine work, another participant stated: "Most of our instructors are occupied with routine work; educating the patients isn't a part of our daily work, so we don't do it, because they don't ask us to do it" (participant No. 16).

Another participant said: "In many wards, we don't have a specific program. In the morning, we go to the ward and start doing routine work. Many instructors just want us to have physical presence in the ward and don't care what we do until noon, and the same goes for patient education" (participant No. 7).

\section{Education environment-related barriers}

Participants described the education environment as another obstacle to patient education. The absence of suitable tools and atmosphere for providing patient education, the high workload, the minimum priority of patient education in the wards, and physicians and nurses' disregard for the issue were cited as the important barriers to patient education. 
One of the participants said: "even if somebody wants to educate the patient, there's no place to do this; there must a place to do this and some tools; maybe a patient doesn't like to be educated about his condition in front of other patients" (participant No. 21).

In regard to the role of education environment as a barrier to patient education, one participant said: "The wards are so busy and the workload is so high that we even struggle to follow our routines and respond to the clinical needs of patients" (participant No. 20).

Another participant stated: "teaching the patient isn't a priority in any ward; so far, I haven't seen any ward putting this in its protocol; even doctors and nurses themselves don't take this seriously" (participant No. 14).

\section{Curriculum-related barriers}

All participants believed that one of the barriers to patient education in the minimum attention to the issue in the nursing education curriculum. Participants stated that during their education, they must pass during their studies a lesson titled "Learning process and principles of patient education." They stated that the issue of patient education is not a priority for the nursing community, and they do not see it as a responsibility of nurses.

In this regard, one of the participants said: "We only had a lesson about patient education, and I don't think that even that was about educating patients. If patient education is so important, why they don't teach us anything about it" (participant No. 21).

Another participant said: "During the six semesters, we have only one short lesson about patient education; so that shows the triviality of this issue; throughout all my semesters, no one in the hospitals have said anything about it; so don't expect this to be important to me" (participant No. 19).

\section{Discussion}

The purpose of this study was to survey the experiences of nursing students about the barriers to patient education in educational medical centers. The themes identified in the remarks of students about this issue were categorized as student-related, patientrelated, instructor-related, education environmentrelated, and curriculum-related barriers.

According to the results, participants believed that there are many challenges ahead of realization of patient education in hospitals. The results of other studies also indicate that nurses and nursing students cannot provide appropriate education to the patient $[7,8,14,16,17]$. In this study, the most important barriers to patient education included the students' lack of motivation and willingness, lack of sufficient academic knowledge, lack of self-confidence, and inability to express academic knowledge in simple words for the patient to understand. Noohi and Abbaszadeh [18] quote Habel as stating that "A large number of clinical nurses have given a vague explanation about the principles of learning and concepts of education, and many do not feel confident or comfortable in regard to their educational skills." The students' inability to provide an understandable scientific explanation to the patients prevents their active participation in patient education and makes them unmotivated and disinterested in regard to the issue. In a study conducted on nursing students, they were found to be reluctant to provide education to the patients [7]. In many studies, the students' lack of readiness, confidence, adequate knowledge, and necessary communication skills have been highlighted as the barriers to patient education $[7,14,18]$. Nursing students need to be informed about the merits and effects of patient education since the very first semesters. In both theoretical and clinical 
classes, instructors must pay more attention to the principles and process of patient education and provide the students with practical training in this regard. Since many students also have part-time jobs, the fatigue may also undermine their willingness to participate in patient education.

In addition to student-related issues, many barriers to patient education seem to be related to patients' unwillingness or disinterest in receiving an education. Patients' lack of demand and participation discourages the students from pursuing this goal. Also, some patients, and especially those with severe and chronic illnesses, are unable to participate in the education. Arian et al. [19] mention the patients' poor education, lack of cooperation, poor health status, and inability in self-care as the obstacles to patient education. Hekari and Mohammadzadeh [16] state that patient education in hospitals is faced with many challenges, one of which is the patients' anxiety and poor physical condition that not only diminish their ability to learn but also prevent them from understanding the impact and importance of education. Tahery et al. [8] also mention the patients' poor physical and mental fitness as a barrier to patient education. In another study, it has been stated that people with low levels of education are less able to acquire the skills needed for self-care, as the ability to acquire these skills is correlated with the level of education [20].

The patients' inability to communicate with nursing students was another important obstacle to the provision of patient education. Participants talked about the cultural differences between the patients and the students, and the refusal of some patients to receive education from students. Also, because of the cultural differences, some patients are unable to or refuse to communicate in any way other than their native language or local dialect. Some participants stated that were not able to understand the language of some patients and had problems in communicating with them. Another barrier to education was the illiteracy of some patients, which caused problems in understanding the scientific explanations given to patients. Considering the importance of patient education, to facilitate the education of illiterate or less educated patients, nurses and nursing students must be provided with visual and auditory tools including educational films, multimedia contents, and graphic posters and at the same time, the challenge of curriculum-related barrier.

Many of the participants stated that many patients have a poor opinion toward the nursing students, and refuse to accept their care and services, even in the presence of an instructor. In a previous study, the distrust between patient and educator has been cited as a barrier to patient education [8]. Also, Ghorbani et al. [7] report that patients do not trust the education provided by nursing students. In general, past studies have reported that the most important barriers to patient education are the patients' refusal to accept education, their inability to receive education, their lack of readiness and motivation, their age, their level of understanding, their emotional and physical condition, severity of their illness, type of their illness (heart disease, surgery, mental illness, etc.) and their distrust towards the ability of nursing students to be a reliable source of education [17,21].

According to the participants, another barrier to patient education is the daily practices and programs of nursing instructors. They stated that many nursing instructors simply ignore the issue when planning the daily schedule. Instructors' inattention to the issue of patient education, their failure to give patient education assignments to the students, their focus on routine clinical works and their emphasis on the physical presence of students in the ward to perform such works, 
their disregard for the importance of patient education, the absence of division of labor, and the lack of a clear daily assignment plan were, in view of participants, the important instructor-related barriers to patient education. In another study, nursing students had stated that providing patient education makes no contribution to their grade and will not be considered in the final evaluation [7]. A study also reported that to provide patient education, nursing students need the support of their instructors throughout the process [17]. It is imperative that nursing instructors pay more attention to patient education in the daily planning of clinical education, and include it in the final evaluation to encourage participation.

The absence of education environment and atmosphere in hospitals was cited as another barrier to patient education. The lack of suitable space equipped with suitable tools for patient education, the high workload, the low priority of patient education in the wards, and the physicians and nurses' disregard for the importance of patient education, were the barriers in this category. Noohi and Abbaszadeh [18] state that in many cases, the poor quality of patient education stems from the patterns of clinical environment. According to Tahery et al. [8], most of the students interviewed in their work believed that the environment, time, and instruments of education are particularly important in the successful implementation of patient education. Citing the findings of Mardanian [22], they add that sufficient instruments and equipment is $92.3 \%$, sufficient time is $73.4 \%$, dedicated space is $49.8 \%$, and dedicated sessions is $95.4 \%$ effective in the successful implementation of patient education [8]. In addition to the mentioned barriers, some studies have reported the absence of proper support, supervision and encouragement for nursing managers, the lack of written educational resources, and the lack of consistent planning as other barriers to patient education [22,23].
Hekari and Mohammadzadeh [16] state that hospitals and medical centers lack the suitable space and equipment for patient education, and cannot provide such education without violating the patient's privacy. Hospital managers can eliminate this environmental barrier by providing the dedicated space and equipment for patient education purposes. Also, nursing managers and head nurses should consider adding the patient education into the list of nursing duties and include it in evaluations as a performance measure.

During their entire education, nursing students encounter the concept of patient education in only a single short lesson named "learning process and principles of patient education." Accordingly, they stated that patient education is not a priority in the nursing education curriculum. Given the low level of importance given to patient education in their curriculum, nursing students do not consider it a part of their duties and do not feel obliged to put any effort in this regard. According to Noohi and Abbaszadeh [18], evaluation of academic performance during an internship period for the "learning process and principles of patient education" lesson will affect students' attitude toward patient education and thereby the structure of patient education in hospitals. Participants of this study also believed that a single lesson cannot provide the nursing students with enough academic and practical skills in the area of patient education and is insufficient for the training of efficient nursing staff for the future. The officials responsible for the planning of nursing education curriculum should, therefore, consider adopting appropriate educational measures to address this issue. Also developing (with other schools) a course that puts more stress on patient education would be one important way to overcome the barriers of patients' education. 


\section{Research limitations}

One of the limitations of this study as a qualitative research is the small number of participants, which restricts the generalizability of the results. Also, the fact that all participants were drawn from the same university limits the transferability of findings. Future works on the subject are recommended to use a larger sample of nursing students drawn from multiple universities to avoid this deficiency. Given the themes identified in this study, future works are suggested to investigate each theme at a greater level of detail.

\section{Conclusion}

The interviewed nursing students highlighted many obstacles to the provision of patient education in hospitals. Some of the barriers were related to the poor academic performance of nursing students. It was found that many patients may not be prepared to receive an education because of age, illiteracy, or cultural and social barriers. Hospital officials can appoint a nurse as the person responsible for providing patient education at appropriate times. Clinical nursing instructors should give a higher priority to patient education and consider using it as a student evaluation criterion in order to encourage more participation on the part of students. Also, patient education practice should be performed in all wards of hospitals by the entire medical staff and a training environment should be provided for this purpose. The officials at the Ministry of Health who are in charge of the nursing education curriculum are recommended to increase the duration and credit of "learning process and principles of patient education" course.

\section{ORCID:}

Mohammad Abbasi: https://orcid.org/0000-0003-1698-5281;

Leili Rabiei: https://orcid.org/0000-0002-5269-2315;

Reza Masoudi: https://orcid.org/0000-0003-3534-0550

Acknowledgements: None.

Funding: None.

Conflicts of interest: No potential conflict of interest relevant to this article was reported.

Author contributions: Conception or design of the work: MA, LR, RM; data collection: MA, RM; data analysis and interpretation: MA, LR, RM; and drafting the article and critical revision of the article, and final approval of the version to be published: MA, LR, RM.

\section{References}

1. Aarabi A, Cheraghi MA, Ghiyasvandian S. Modification of nursing education for upgrading nurses' participation: a thematic analysis. Glob J Health Sci. 2015;7(4):161172.

2. Kashani F, Moghimian M, Salarvand S, Kashani P. Nurses' knowledge, attitude, practice about effective communication skills in patient education. J Res Dev Nurs Midwifery. 2015-2016;12(2):59-67.

3. Mousavi MS, Taghadosi M, Rezaie Shahsavarloo Z. Nursing staff views about the barriers to patient education and provided strategies to improve it in hospitals affiliated to Kashan University of Medical Sciences, 1391. J Urmia Nurs Midwifery Fac. 2014;12(9):842-849.

4. Marcum J, Ridenour M, Shaff G, Hammons M, Taylor M. A study of professional nurses' perceptions of patient education. J Contin Educ Nurs. 2002;33(3):112-118.

5. Strömberg A. The crucial role of patient education in heart failure. Eur J Heart Fail. 2005;7(3):363-369.

6. Dehghani A, Orang M, Abdollahi FS, Parvianian NA, 
Vejdani M. Barriers to patient education in clinical care; viewpoints of nurses. Iran J Med Educ. 2014;14(4):332341.

7. Ghorbani R, Soleimani M, Zeinali MR, Davaji M. Iranian nurses and nursing students' attitudes on barriers and facilitators to patient education: a survey study. Nurse Educ Pract. 2014;14(5):551-556.

8. Tahery N, Razavi Zadegan M, Kaiali M, et al. Importance of patient education according Abadan nursing students opinions. Mod Care J. 201 1;8(2):100-106.

9. Edwardson SR. Patient education in heart failure. Heart Lung. 2007;36(4):244-252.

10. Deccache A, Aujoulat I. A European perspective: common developments, differences and challenges in patient education. Patient Educ Couns. 2001;44(1):7-14.

11. Rostami H, Montazam SH, Ghahremanian A. Survey of education barriers from nurses and patients viewpoint. Sci J Hamadan Nurs Midwifery Fac. 2010;18(1):50-60.

12. Heidari MR, Norouzadeh R. Nursing students' perspectives on clinical education. J Adv Med Educ Prof. 2015; 3(1):39-43.

13. Abbaszade A, Borhani F, Sabzevari S. Nursing teachers perception of the challenges of clinical education and solutions: a qualitative study. J Qual Res Health Sci. 2013;2(2):134-145.

14. Jahromi ZB. A study of the barriers and facilitators of patient education from the viewpoint of nursing students at Jahrom College of Nursing. Bangladesh J Med Sci. 2016;5(3):471-476.

15. Speziale HS, Streubert HJ, Carpenter DR. Qualitative research in nursing: advancing the humanistic imperative. 5th ed. Philadelphia, USA: Lippincott Williams \& Wilkins; 2011.
16. Hekari D, Mohammadzadeh R. Quality of patients' education by nursing students and employment nurses and its effective factors in Tabriz hospitals in 2007-2008. Med Sci J Islamic Azad Univ Tehran Med Branch. 2010;20(1):58-63.

17. Choi WH, Hui GK, Lee AC, Chui MM. Student nurses' experiences and challenges in providing health education in Hong Kong. Nurse Educ Today. 2010;30(4):355-359.

18. Noohi E, Abbaszadeh A. Process of patient education and Orem's self care theory, an integrative model curriculum: a qualitative study. J Qual Res Health Sci. 2017; 5(4):419-431.

19. Arian M, Mortazavi H, TabatabaeiChehr M, Tayebi V, Gazerani A. The comparison between motivational factors and barriers to patient education based on the viewpoints of nurses and nurse managers. J Nurs Educ. 2015;4(3):66-77.

20. Jaarsma T, Cameron J, Riegel B, Stromberg A. Factors related to self-care in heart failure patients according to the middle-range theory of self-care of chronic illness: a literature update. Curr Heart Fail Rep. 2017;14(2):7177.

21. Schyve PM. Language differences as a barrier to quality and safety in health care: the Joint Commission perspective. J Gen Intern Med. 2007;22 Suppl 2:360-361.

22. Mardanian L. Assessing the nurses perception about patient training in Isfahan University of Medical Sciences. Isfahan Univ Med Sci. J 2007;17(25):18-40.

23. Sultani A, Hadavi M, Heydari S, Shahabinezhad M. Barriers to patient education based on the viewpoints of nurses and nurse managers in hospitals of Rafsanjan University of Medical Sciences, 2008. J Rafsanjan Univ Med Sci. 2013;12(4):309-318. 